\title{
The distances of planetary nebulae: A scaling factor based upon radial velocities
}

\author{
J. P. Phillips \\ Instituto de Astronomia y Meteorologia, Avenida Vallarta 2602, Col. Arcos Vallarta, CP 44130 Guadalajara, \\ Jalisco, Mexico
}

Received 19 May 2000 /Accepted 1 December 2000

\begin{abstract}
We have used the observed radial velocities of planetary nebulae, and the galactic rotation curve to define a new distance scale for planetary nebulae. Care has been taken to restrict the analysis to sources in which distance non-linearities are minimised, and for which distortion of the distance scale is likely to be small. We conclude, as a result, that the so-called "long" distance scales are the most valid, and that various "shorter" scales are unlikely to be correct. The primary systematic errors in this procedure derive from uncertainties in the galactic rotation profile, and in $\Theta_{0}$ and $R_{0}$. Such errors are less than those normally associated with PN distance determinations.
\end{abstract}

Key words. planetary nebulae: general - Galaxy: kinematics and dynamics

\section{Introduction}

The rotational profile $\Theta(R)$ of the Galaxy has been measured using the radial velocities and distances of a variety of sources, including $\mathrm{HI}$ and HII regions (Burton \& Gordon 1978; Blitz et al. 1982; Fich 1989; Brand \& Blitz 1993), CO clouds (Blitz et al. 1982; Clemens 1985), OB stars and classical Cepheids (Fich et al. 1989; Frink et al. 1996; Pont Mayor \& Burki 1994), OH/IR stars (Amaral et al. 1996), young open clusters (Hron 1987) and carbon stars (Metzger \& Schechter 1994). Excepting for the cases of the CO/HI/HII regions, where $\Theta(R)$ is determined using tangential velocities, it usually preferable to select sources for which distances are well known, and whose velocity dispersions are small. Neither of these constraints is true for planetary nebulae $(\mathrm{PN})$, although these sources have, nevertheless, also been used to evaluate $\Theta(R)$ (e.g. Berman 1937; Dutra \& Maciel 1990; Amaral et al. 1996).

We note for instance that there are at least two PN distance scales doing the rounds at present, one (the "short" scale) based upon the work of O'Dell (1962), Daub (1982) and others, the other ("long") scale deriving from the analyses of Mendez et al. (1988), Mallik \& Peimbert (1988) and Peimbert (1990). These have co-existed over several years, with little evidence for a coming together - or of clear evidence as to which is correct.

Send offprint requests to: J. P. Phillips, e-mail: jpp@udgserv. cencar.udg.mx
Apart from this, errors and uncertainties in nebular characteristics are usually such as to create (nonsystematic) uncertainties in distance of at least $30 \%$, whatever procedure is chosen to evaluate this parameter.

The radial velocities of $\mathrm{PN}$ are also unreliable, in the sense that most PN within the galactic disk (i.e. older population I type sources) show a velocity dispersion of $\sim \pm 40 \mathrm{~km} \mathrm{~s}^{-1}$ - that is, their peculiar velocities with respect to their LSRs have a typical range $\sim 80 \mathrm{~km} \mathrm{~s}^{-1}$ (Pottasch 1984). The population II sources towards the galactic centre are even less viable in this respect, since the dispersion in their velocities is $\sim 140 \mathrm{~km} \mathrm{~s}^{-1}$, and there is significant evidence for non-circular motion (see for instance the discussion of Pottasch 1984).

It appears, therefore, that measurements of such sources are likely to have only limited utility in helping to define the galactic rotation curve (although see also Schneider \& Terzian 1983). On the other hand, it is clear that since the rotation curve of the Galaxy is tolerably well known, particularly over galacto-centric distances $R<R_{0}$ (where $R_{0}=8.5 \mathrm{Kpc}$ is the IAU-approved value for the Sun's distance from the galactic centre), then radial velocities may be used to tie down the PN distance scale or at least, they may be used to do so within the accuracy to which galactic rotational parameters are known. Of course, the large peculiar velocities of PN mean that no individual distance can be considered as reliable. However, a statistical analysis of many such sources may, in principal, be used to constrain mean distances. 
We shall be assuming, for the purpose of this analysis, that the peculiar velocities of population I disc PN display no orientational preference - that is, that there is no galactic streaming of the $\mathrm{PN}$ population. This, so far as we can assess, appears consistent with observed nebular dynamics (Pottasch 1984).

We shall find that a careful evaluation of $\mathrm{PN}$ radial velocities implies a distance scale on the "long" side - very similar, in fact, to the recent statistical distance scales of van de Steene \& Zijlstra (1994) and Zhang (1995). The shorter distance scales are almost certainly in error.

\section{Evaluation of nebular distances}

For the purposes of this analysis, we shall presume that PN velocities partake of circular galactic rotation, and that the rotational profile $\Theta(R)$ of the Galaxy is given through the published curves of Clemens (1985) and Burton \& Gordon (1978) (both are almost identical over the radial range $R<R_{0}$ of interest to this analysis). We shall approximate such trends using a sixth order least squares polynomial regression curve. Under these circumstances, the radial velocities of the sources are given through (Schneider \& Terzian 1983)

$V_{\mathrm{r}}=\left(\Theta(R)\left(R_{0} / R\right)-\Theta_{0}\right) \sin l \cos b$,

where $l$ and $b$ are the galactic longitude and latitude of the source, $\Theta(R)$ is the LSR velocity at distance $R$ of the source from the galactic centre, and $\Theta_{0}$ corresponds to the LSR velocity at distance $R=R_{0}$. Finally, $R$ is the distance of the source from the galactic centre projected onto the plane, given through

$R=\left(R_{0}^{2}+\left(D_{\mathrm{N}} \cos b\right)^{2}-2 R_{0} D_{\mathrm{N}} \cos b \cos l\right)^{0.5}$,

where $D_{\mathrm{N}}$ is the distance of the source from the Sun in the near part of the galactic plane (i.e. for values $D_{\mathrm{N}}<R_{0}$ $\cos l$ where $\left.b=0^{\circ}\right)$. Equivalent velocities are also obtained for distances $D_{\mathrm{F}}=\left[\left(2 R_{0} \cos l-D_{\mathrm{N}} \cos b\right) \sec b\right]$ on the far side of the plane, although this ambiguity causes very little problem in the proceeding analysis. Comparison of distances derived here with those evaluated by Zhang (1995) suggest that only $\sim 10 \%$ of sources in the present sample lie on the farther side of the galactic disk.

Because of the complex nature of the function $\Theta(R)$, the distances $D_{\mathrm{F}}$ and $D_{\mathrm{N}}$ are most readily evaluated using numerical procedures. A careful study of these solutions suggest that there is no ambiguity in $D$ for the present source sample - apart, that is, from the values $D_{\mathrm{N}}$ and $D_{\mathrm{F}}$ noted above.

Before proceeding to an analysis of individual source distances, it is necessary to select an appropriate sample of radial velocities; not all of the more that 900 published values will serve to determine such distances.

\section{Constraints upon the radial velocity sample}

We impose several restrictions upon the selection of sources for this analysis: a) Sources are excluded if they possess galactic latitudes $0<l<10^{\circ}$, and $350^{\circ}>l>360^{\circ}$; thereby eliminating the large majority of population II PN (bulge sources) which do not follow the disk rotation curve;

b) We also exclude sources having $90^{\circ}<l<270^{\circ}$, since the galactic rotation curve is relatively ill-defined for radii $R>R_{0}$ (see for instance Honma \& Sofue 1997);

c) We only include nebulae possessing galactic latitudes $|b|<15^{\circ}$, in order to restrict the sample to those sources which are clearly participating in disk rotation. In fact, of the sample to be analysed later, fully $90 \%$ have latitudes $|b|<10^{\circ}$;

d) Finally, and perhaps most importantly, we select ranges of $l$ and $V_{\mathrm{r}}$ over which non-linearities in the distance scale are likely to be small. In particular, we eliminate sources having $V_{\mathrm{r}}<V_{\mathrm{A}}=40 \mathrm{~km} \mathrm{~s}^{-1}$, and which have velocities within $40 \mathrm{~km} \mathrm{~s}^{-1}$ of the maximum (tangential) rotational velocity along any particular longitude $l$. Specifically:

$$
V_{\mathrm{r}} \leq V_{\mathrm{B}}=\Theta\left(R=R_{0} \sin (l)\right)-\Theta_{0} \sin (l)-40 \mathrm{~km} \mathrm{~s}^{-1} .
$$

Assuming to a first approximation that $\Theta(R) \approx$ $\Theta\left(R_{0}\right)=220 \mathrm{~km} \mathrm{~s}^{-1}$, this then yields

$V_{\mathrm{r}}<(180-220 \sin (l)) \mathrm{km} \mathrm{s}^{-1}$,

suggesting that $l<40^{\circ}$ where $V_{\mathrm{r}}>40 \mathrm{~km} \mathrm{~s}^{-1}$. Given the constraint cited in (a) above, this then implies that only sources having $10^{\circ}<l<40^{\circ}$, and $320^{\circ}<l<350^{\circ}$ are appropriate for inclusion in this analysis.

This last point requires some further explication. It is important, for the purposes of this analysis, to note that there is a non-linear relationship between radial velocity $V_{\mathrm{r}}$ and the associated distance $D$ - a doubling in $V_{\text {r }}$ does not necessarily imply a corresponding doubling in distance. Thus, an ensemble of PN having identical distances $D_{\mathrm{s}}$ and galactic longitudes $l$ may, nevertheless, appear to possess a skewed distribution of distances where $D$ is calculated through the procedure outlined in Sect. 2, and the sources possess a symmetric Gaussian velocity dispersion $\sigma_{\mathrm{v}}$ along the line of sight. This tendency is illustrated in Fig. 1, where we show two cases corresponding to differing values of $D_{\mathrm{s}}$, and differing values of galactic longitude. We have also indicated the actual distances $D_{\mathrm{s}}$ of these sources by vertical lines - the scatter of distances either side of these lines representing an artefact of the velocity dispersion. It is clear from this that although the radial velocity distribution may peak symmetrically about $V_{\mathrm{s}}$ (the velocity corresponding to galactic rotation at the position of the ensemble), the distance function which is actually "measured", using the procedures of Sect. 2, peaks at a value $D_{\text {peak }}>D_{\text {s }}$. Furthermore, the apparent mean distance $\langle D\rangle$ of this particular ensemble will exceed the actual distance $D_{\mathrm{s}}$ - there will be a scaling error $\langle D\rangle / D_{\mathrm{s}}$. This scaling error may, taken more generally, have a value which is greater or less than unity.

The scaling error is illustrated in Fig. 1 for the case where $l=10^{\circ}$ and $V_{\mathrm{s}}=50 \mathrm{~km} \mathrm{~s}^{-1}$, where it may be seen 


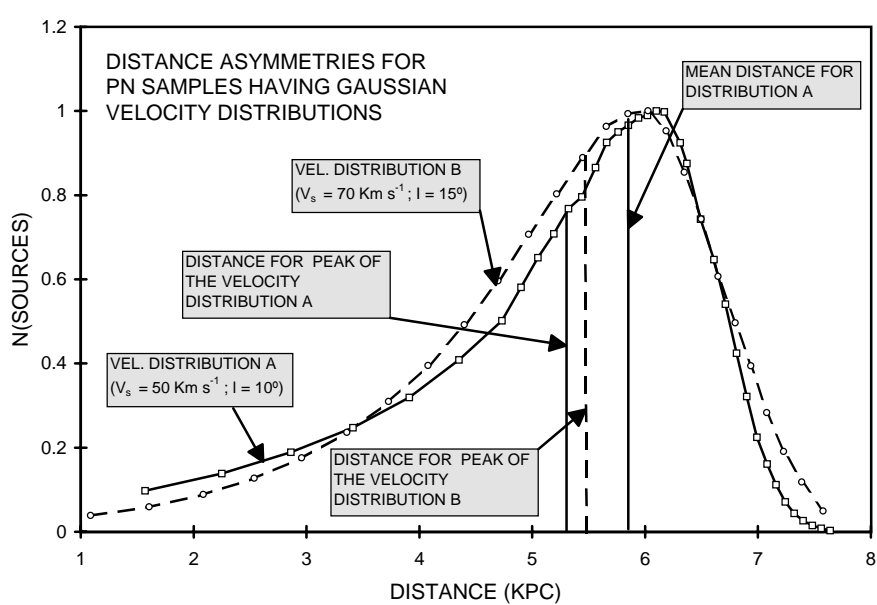

Fig. 1. The normalised distributions of nebular distances which would be deduced for sources located at the same distance $D_{\mathrm{s}}$, and having identical longitudes $l$, but which possess a symmetric and Gaussian distribution of velocities about the mean radial velocity $V_{\mathrm{s}}$ (corresponding to a velocity dispersion of $40 \mathrm{~km} \mathrm{~s}^{-1}$ ). Two cases are illustrated: velocity distribution A (in which $V_{\mathrm{s}}=50 \mathrm{~km} \mathrm{~s}^{-1}$ and $l=10^{\circ}$ ), and velocity distribution B (for which $V_{\mathrm{s}}=70 \mathrm{~km} \mathrm{~s}^{-1}$ and $l=15^{\circ}$ ). The galactic latitudes are assumed in both cases to be zero

that the mean velocity $\langle D\rangle$ (indicated by the right hand vertical line) has a value greater than $D_{\mathrm{s}}$ (the left hand vertical line), the actual distance to this ensemble of sources.

A little thought shows that where the velocity dispersion of the sources is $\sigma_{\mathrm{v}}=40 \mathrm{~km} \mathrm{~s}^{-1}$, then the asymmetries will become more pronounced as $\left|V_{\mathrm{s}}\right|$ descends below $40 \mathrm{kms}^{-1}$; not least because more and more sources will appear to have positive velocities where $0>\left(V_{\mathrm{s}} / \mathrm{km} \mathrm{s}^{-1}\right)$ $>-40$, or negative velocities where $0<\left(V_{\mathrm{s}} / \mathrm{km} \mathrm{s}^{-1}\right)<40$. Thus, in any ensemble of nebulae whose actual distance is $D_{\mathrm{s}}$; for which $V_{\mathrm{s}}$ is positive and less than $40 \mathrm{~km} \mathrm{~s}^{-1}$; and for which the velocity dispersion is $\sigma_{\mathrm{v}}=40 \mathrm{~km} \mathrm{~s}^{-1}$, then many of the nebulae will appear to have negative radial velocities. It would be impossible, for these sources, to employ the procedures of Sect. 2. The nebulae at the lower end of the dispersion function essentially fall-out of the analysis, leaving only a diminished rump of sources with which to calculate $\langle D\rangle$. $\langle D\rangle$ will, in consequence, appear larger than $D_{\mathrm{s}}$. It is for this reason that we have assigned a velocity constraint $V_{\mathrm{r}}<V_{\mathrm{A}}=40 \mathrm{~km} \mathrm{~s}^{-1}$ in subsection (d) above. A similar argument applies for sources within $40 \mathrm{~km} \mathrm{~s}^{-1}$ of the maximum (tangential) rotational velocity.

The variation in the ratio $\left\langle D>/ D_{\mathrm{s}}\right.$ is illustrated in Fig. 2 for a range of values $l$ and $V_{\mathrm{s}}$ corresponding to the constraints indicated in subsection (d), and assuming $b=0^{\circ}$. Similar values of $\langle D\rangle / D_{\mathrm{s}}$ will arise for whatever value of $|b|<15^{\circ}$ is assumed. Note, again, that $V_{\mathrm{s}}$ corresponds to the radial velocity which the sources would have were there no dispersion in velocities $\sigma_{\mathrm{v}}$ (i.e. $\sigma_{\mathrm{v}}=0$ ), and the distance was $D=D_{\mathrm{s}}$, whilst $\langle D\rangle / D_{\mathrm{s}}$ measures the skewness described above, arising from the fact that

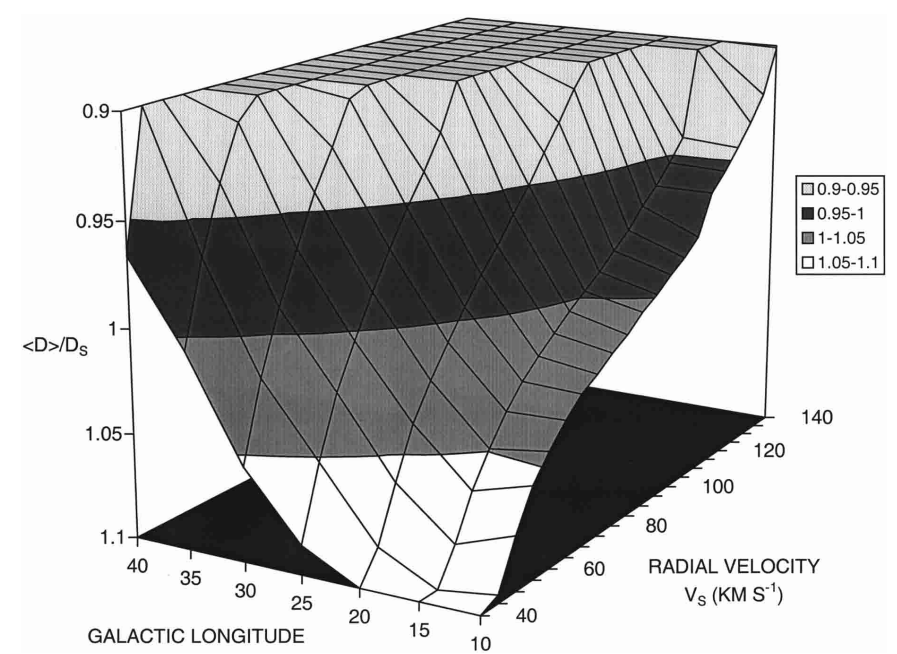

Fig. 2. The variation of $\langle D\rangle / D_{\mathrm{s}}$ with galactic longitude $l$ and radial velocity $V_{\mathrm{s}}$ (see text for details). The range of parameters is selected so as to minimise distance asymmetries, and yield reduced values of $\langle D\rangle / D_{\mathrm{s}}$. These ranges of parameter are also used to evaluate the distance scales in Table 1

$\sigma_{\mathrm{v}}$ is in fact non-zero. In addition, however, the diagram represents a useful guide to the ranges of longitude and radial velocity over which nebulae should be selected. Where $|\langle D\rangle| D_{\mathrm{s}} \mid$ is appreciably different from unity for any particular combination of $l$ and $V_{\mathrm{s}}$, then nebulae having similar values of longitude and radial velocity $V_{\mathrm{r}}$ should almost certainly be excluded from this analysis; they would be prone to cause substantial scaling errors.

It is in fact plain, in Fig. 2, that the ratios $\langle D\rangle / D_{\mathrm{s}}$ take values which are reasonably modest $(0.90<<D>/$ $\left.D_{\mathrm{s}}<1.15\right)$, whilst an analysis of values over the entire map indicates an overall mean $\left\langle\left[\langle D\rangle / D_{\mathrm{s}}\right]\right\rangle \sim 0.99$. It is clear, therefore, that where sources occupy the $l-V_{\mathrm{r}}$ regime of subsection (d) in a uniform manner, then biases in mean distance $\left\langle D>=\sum_{i=1}^{n} D_{i} / n\right.$ will be small to negligible. Where the distribution of sources is not uniform within this plane (and this will normally be the case), then it is apparent that errors in $\langle D\rangle$ may be larger, although they are unlikely to exceed a few percent.

Broadly speaking, therefore, it would seem that if the mean distance is evaluated to nebulae having the radial velocity, longitude, and latitude ranges described above (subsection (d)), using the procedures outlined in Sect. 2, then this value should prove reasonably accurate.

Care must however be taken not to exceed these constraints. The dangers of so doing are illustrated in Fig. 3, where we show how $\langle D\rangle / D_{\mathrm{s}}$ varies for a broader range of velocities $V_{\mathrm{S}}$ than that illustrated in Fig. 2. It is plain from this that as $V_{\mathrm{s}}$ becomes larger than $V_{\mathrm{B}}$ (see subsection (d) above), or less than $V_{\mathrm{A}}$, then $|\langle D\rangle| D_{\mathrm{s}} \mid$ may become unacceptably large; a situation which again implies that individual sources with radial velocities $V_{\mathrm{r}}>V_{\mathrm{B}}$, or $V_{\mathrm{r}}<V_{\mathrm{A}}$ are best left out of the analysis. There would be a danger of finding mean distances which are unacceptably large or small compared to the actual mean distances. 


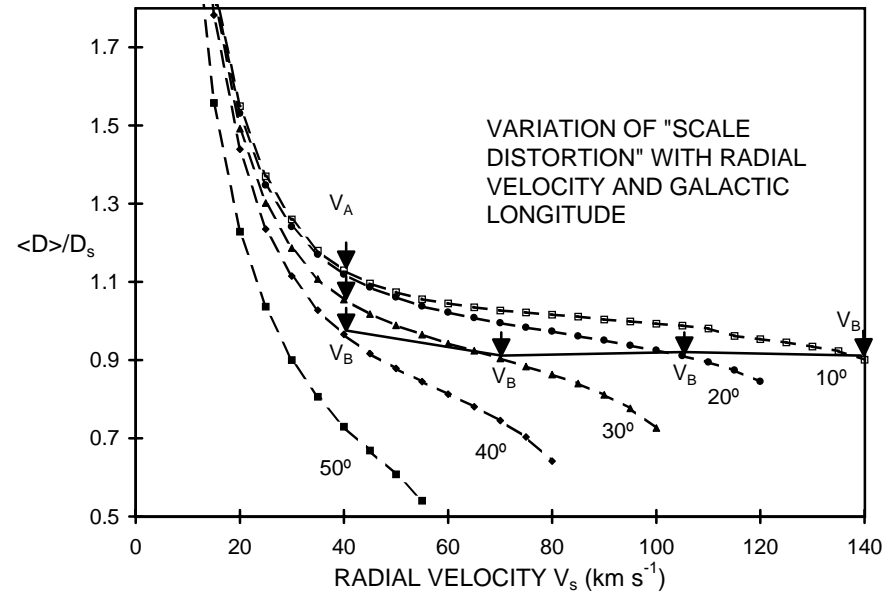

Fig. 3. Variation of $\langle D\rangle / D_{\mathrm{s}}$ with $V_{\mathrm{s}}$ for five values of galactic longitude and $b=0^{\circ}$. The ratio is calculated for a broader range of velocities than Fig. 2, and shows the tendency for $\langle D\rangle / D_{\mathrm{s}}$ to become rapidly larger or smaller at small and large values of $V_{\mathrm{s}} . V_{\mathrm{A}}$ and $V_{\mathrm{B}}$ correspond to limiting radial velocities (see text for details), whilst the numbers associated with the individual curves correspond to values of galactic longitude

This has, to a certain degree, been a rather complex discussion. The conclusions however are quite straightforward. It would seem that providing the constraints described above are rigorously enforced, then the mean distances of PN should be reliable; errors in the deduced distance scale, or arising from ambiguities in distance should be comparatively small.

\section{The distance scale of planetary nebulae}

As we have described above, the individual distances of PN (derived using the procedures described in Sect. 2) will be exceedingly unreliable - a consequence of the large dispersion in the peculiar velocities of these sources. However, it is possible (given a large enough sample) to evaluate a mean distance for the nebulae, and to compare this with published distances in order to evaluate relative distance scales. The constraints noted in Sect. 3 (in combination with the velocities catalogued by Durand et al. 1998; Schneider et al. 1983; Acker et al. 1992) result in a sample of only 49 sources, although we shall find that this is sufficient to arrive at statistically interesting conclusions concerning the distances to $\mathrm{PN}$ in general. Details of the sources, of their radial velocities, deduced distances $\left(D_{\mathrm{N}}\right.$ and $D_{\mathrm{F}}$; see Sect. 2), and associated values of $R$ and $\Theta(R)$ are given in Table 1.

We have compared the present distances to those published by Zhang (1995); van de Steene \& Zijlstra (1994); Cahn et al. (1992); Cahn \& Kaler (1971); Acker (1978) and Daub (1982), and evaluated a scaling parameter $\Gamma=<D$ (Reference Sources) $>/<D(\mathrm{US})>$ for each case. The results are summarised in Table 2 , where $N$ is the number of sources common to both the present sample and the referenced results.
It is plain from this that the distance scale derived here is very closely similar to those deduced by Zhang (1995) and van de Steene \& Zijlstra (1994); although note that the latter authors employ a solar galacto-centric distance $R_{0}$ of $8.0 \mathrm{kpc}$. Where this is increased to $R_{0}=8.5 \mathrm{kpc}$, the value employed here, then the scale values in Table 2 should be increased by $6.25 \%$. Our present distance scale, on the other hand, is very much larger than those of Acker (1978), Cahn \& Kaler (1971) and Daub (1982), whilst the distance scale of Cahn et al. (1992) appears to be intermediate between those of Daub (1982) and ourselves. Given the relation between various other distance scales and that of Daub (see e.g. Kingsburgh \& Barlow 1992), it is clear that the scale derived here is also comparable to those of Cudworth (1974) and Kingsburgh \& Barlow (1992), but is significantly larger than those of Maciel (1984), Maciel \& Pottasch (1980), Milne (1982), and O'Dell (1962).

We therefore conclude that the distances of PN based on observed radial velocities, and the deduced galactic rotation curve, are most consistent with the "long" distance scale. It is unlikely that this is any appreciable way biased through the distance asymmetries described in Sect. 3. Nor, similarly, is it likely that uncertainties in galactic rotation will greatly affect these results. Thus for instance, although the parameter $R_{0}$ is open to some uncertainty, this is unlikely to exceed $\sim 6 \%$ (see the various estimates of this parameter summarised by Reid 1993; Fich \& Tremaine 1991). Similarly, estimates of $\Theta_{0}$ vary from $\sim 180 \mathrm{~km} \mathrm{~s}^{-1}$ (Rohlfs et al. 1986; Rohlfs \& Kreitschmann 1987; Kuijken \& Tremaine 1994) through to $\sim 200 \mathrm{~km} \mathrm{~s}^{-1}$ (Merrifield 1992; Honma \& Sofue 1997). Frink et al. (1996), by contrast, appear to derive values of $\Omega_{0}$ some $\sim 13 \%$ greater than the standard IAU value.

The situation is therefore far from clear, although if (as the overall trends seem to suggest) $R_{0} \cong 8.0 \mathrm{kpc}$ and $\Theta_{0} \cong 190 \mathrm{~km} \mathrm{~s}^{-1}$, then our distance scale would require increasing by only $\sim 2 \%$ (after appropriate modification of the trends of Clemens 1985; Burton \& Gordon 1978). It appears that the changes in $R_{0}$ and $\Theta_{0}$ cancel each other to a surprisingly close degree.

Finally, we note that Schneider \& Terzian (1983) have also used $\mathrm{PN}$ radial velocities to evaluate the distance scale to these sources, although they use a significantly different procedure to that outlined above. In particular, these authors plot radial velocity against distances taken from Acker (1978) for 31 sources close to $l=35^{\circ}$ and $325^{\circ}$. The Acker distances are then proportionately increased, until the observations are approximately in agreement with the trends expected for galactic rotation. The resulting, revised distances imply a scale $\sim 25 \%$ smaller than that determined here. Some of this disparity with the present scale may arise as a result of distance asymmetries in the data of Schneider \& Terzian (see the discussion in Sect. 3).

\section{Conclusions}

The uncertainties in planetary nebula distances have long been notorious, and there have historically been at least 
Table 1. Orbital parameters and distances for selected planetary nebulae

\begin{tabular}{|c|c|c|c|c|c|c|}
\hline$\overline{P G}$ & NAME & $\begin{array}{l}V_{\text {Isr }} \\
\mathrm{km} \mathrm{s}^{-1}\end{array}$ & $\begin{array}{l}D_{N} \\
k p c\end{array}$ & $\begin{array}{l}D_{F} \\
k p c\end{array}$ & $\begin{array}{l}\mathrm{R} \\
\mathrm{kpc}\end{array}$ & $\begin{array}{l}\Theta(R) \\
\mathrm{km} \mathrm{s}^{-1}\end{array}$ \\
\hline $11.1+11.5$ & M 2-13 & 100.0 & 6.91 & 11.00 & 2.27 & 199.8 \\
\hline $13.0-04.3$ & Pe 2-14 & 101.0 & 6.66 & 9.95 & 2.52 & 198.5 \\
\hline $14.4-06.1$ & SB 19 & 84.9 & 6.12 & 10.44 & 3.01 & 199.3 \\
\hline $15.4-04.5$ & M 1-53 & 76.3 & 5.69 & 10.75 & 3.38 & 202.1 \\
\hline $15.6-03.0$ & A 44 & 57.6 & 4.77 & 11.63 & 4.11 & 210.3 \\
\hline $16.0-07.6$ & SB 21 & 79.0 & 5.79 & 10.69 & 3.37 & 202.0 \\
\hline $16.4-01.9$ & M 1-46 & 43.2 & 3.75 & 12.57 & 5.02 & 220.1 \\
\hline $17.5-07.4$ & SB 23 & 65.3 & 4.99 & 11.36 & 4.06 & 209.6 \\
\hline $17.9-04.8$ & M 3-30 & 84.8 & 5.82 & 10.41 & 3.47 & 203.0 \\
\hline $18.0+03.6$ & M 4-8 & 43.4 & 3.57 & 12.63 & 5.22 & 221.8 \\
\hline $19.2-02.2$ & M 4-10 & 64.4 & 4.69 & 11.38 & 4.36 & 213.1 \\
\hline $19.4-05.3$ & M 1-61 & 43.9 & 3.46 & 12.64 & 5.37 & 222.9 \\
\hline $19.7-04.5$ & M 1-60 & 90.2 & 5.93 & 10.12 & 3.55 & 203.7 \\
\hline $19.9+00.9$ & M 3-53 & 49.9 & 3.76 & 12.23 & 5.13 & 221.0 \\
\hline 20.7-05.9 & Sa 1-8 & 47.7 & 3.59 & 12.41 & 5.32 & 222.5 \\
\hline $22.0-03.1$ & M 1-58 & 74.8 & 4.99 & 10.80 & 4.31 & 212.5 \\
\hline $23.3-07.6$ & MaC 1-16 & 68.3 & 4.60 & 11.15 & 4.67 & 216.7 \\
\hline $24.2-05.2$ & M 4-11 & 57.5 & 3.87 & 11.70 & 5.23 & 221.8 \\
\hline $27.3-03.4$ & A 49 & 43.6 & 2.86 & 12.27 & 6.11 & 226.4 \\
\hline $27.4-03.5$ & VY 1-4 & 70.4 & 4.42 & 10.70 & 5.01 & 220.0 \\
\hline $28.0+10.2$ & WeSb 3 & 66.2 & 4.26 & 10.99 & 5.19 & 221.5 \\
\hline $28.5+05.1$ & K 3-2 & 58.8 & 3.71 & 11.29 & 5.54 & 224.0 \\
\hline $31.0-10.8$ & M 3-34 & 52.0 & 3.31 & 11.52 & 5.95 & 225.9 \\
\hline $32.7-02.0$ & M 1-66 & 44.4 & 2.71 & 11.60 & 6.39 & 227.0 \\
\hline $33.8-02.6$ & NGC 6741 & 57.8 & 3.49 & 10.65 & 5.93 & 225.8 \\
\hline $35.9-01.1$ & Sh 2-71 & 41.7 & 2.5 & 11.27 & 6.64 & 227.3 \\
\hline $37.8-06.3$ & NGC 6790 & 56.8 & 3.47 & 10.04 & 6.15 & 226.5 \\
\hline $325.4-04.0$ & He 2-141 & -46.4 & 2.82 & 11.21 & 6.39 & 227.0 \\
\hline $327.8-07.2$ & He 2-163 & -45.0 & 2.81 & 11.69 & 6.32 & 226.9 \\
\hline $331.1-05.7$ & PC 11 & -50.8 & 3.27 & 11.80 & 5.83 & 225.4 \\
\hline $332.9-09.9$ & He 3-1333 & -64.4 & 4.2 & 11.16 & 5.17 & 221.4 \\
\hline $333.4+01.1$ & He 2-152 & -60.7 & 3.89 & 11.31 & 5.32 & 222.5 \\
\hline $334.8-07.4$ & SaSt 2-12 & -60.9 & 4.04 & 11.47 & 5.16 & 221.3 \\
\hline $336.2+01.9$ & $\mathrm{Pe} 1-6$ & -72.2 & 4.72 & 10.84 & 4.60 & 215.9 \\
\hline $336.2-06.9$ & PC 14 & -46.7 & 3.29 & 12.38 & 5.67 & 224.7 \\
\hline $336.3-05.6$ & He 2-186 & -64.2 & 4.31 & 11.33 & 4.89 & 218.8 \\
\hline $340.8+12.3$ & Lo 11 & -72.6 & 5.33 & 11.10 & 3.97 & 208.5 \\
\hline $342.8-06.6$ & Cn 1-4 & -83.3 & 5.85 & 10.50 & 3.41 & 202.4 \\
\hline $343.7-09.6$ & SB 29 & -83.5 & 6.00 & 10.55 & 3.27 & 201.1 \\
\hline $344.2-01.2$ & H 1-6 & -45.2 & 3.98 & 12.38 & 4.80 & 217.9 \\
\hline $345.0-04.9$ & Cn 1-3 & -72.4 & 5.59 & 10.89 & 3.44 & 202.6 \\
\hline $345.2-08.8$ & Tc 1 & -77.9 & 5.9 & 10.73 & 3.23 & 200.8 \\
\hline $346.0+08.5$ & He 2-171 & -75.5 & 5.89 & 10.79 & 3.18 & 200.4 \\
\hline $346.2-08.2$ & IC 4663 & -46.6 & 4.47 & 12.21 & 4.33 & 212.9 \\
\hline $347.4+05.8$ & H 1-2 & -94.4 & 6.55 & 10.13 & 2.57 & 198.4 \\
\hline $347.7+02.0$ & Vd 1-8 & -109.7 & 6.89 & 9.73 & 2.30 & 199.5 \\
\hline $348.0-13.8$ & IC 4699 & -118.6 & 7.36 & 9.76 & 2.12 & 201.2 \\
\hline 349.3-01.1 & NGC 6337 & -66.7 & 5.90 & 10.81 & 2.92 & 198.8 \\
\hline
\end{tabular}


Table 2. Relative distance scales

\begin{tabular}{lll}
\hline GAMMA & $\mathrm{N}$ & REFERENCE DISTANCE SCALE \\
\hline \hline $1.011 \pm 0.122$ & 30 & ZHANG (1995) \\
$1.010 \pm 0.131$ & 25 & VAN DE STEENE \& ZIJLSTRA (1994) \\
$0.830 \pm 0.107$ & 29 & CAHN ET AL. (1992) \\
$0.735 \pm 0.113$ & 21 & CAHN \& KALER (1971) \\
$0.712 \pm 0.099$ & 17 & ACKER (1978) \\
$0.542 \pm 0.089$ & 16 & DAUB (1982) \\
\hline
\end{tabular}

two sets of distances to these sources, differing by a factor $\sim 2$. We have shown, in the present analysis, that a careful consideration of PN radial velocities, allied to the known characteristics of galactic rotation can be used to provide a further constraint upon nebular distances. It is found, in consequence, that the so-called "long" distance scales (i.e. those of Cudworth 1974; Zhang 1995 and others) are the more valid.

There seems little reason to doubt the reliability of the present scale. We have, for instance, been careful to analyse the influence of non-linearities upon the estimate of distance, and have strongly restricted the sample of sources in order to minimise such effects; only 49 sources (out of the more than 900 sources for which measurements exist) were deemed appropriate for inclusion in the present study. Similarly, although the properties of galactic rotation are by no means completely assured, we have employed a range of galactic longitudes and radial velocities over which the galactic rotation curve is best defined. Errors in $R_{0}$ and $\Theta_{0}$ are unlikely to modify the deduced distance scale by more than a few percent.

\section{References}

Acker, A. 1978, A\&AS, 33, 367

Acker, A., Ochsenbein, F., Stenholm, B., et al. 1992, Strasbourg-ESO Catalogue of Planetary nebulae, ESO

Amaral, L. H., Ortiz, R., Lépine, J. R. D., \& Maciel, W. J. 1996, MNRAS, 281, 339

Berman, L. 1937, PASP, 49, 13

Blitz, L., Fich, M., \& Stark, A. A. 1982, ApJS, 49, 183

Brand, J., \& Blitz, L. 1993, A\&A, 275, 67

Burton, W. B., \& Gordon, M. A. 1978, A\&A, 63, 7

Cahn, J. H., \& Kaler, J. B. 1971, ApJS, 22, 319
Cahn, J. H., Kaler, J. B., \& Stanghellini, L. 1992, A\&AS, 94, 399

Clemens, D. P. 1985, ApJ, 295, 422

Cudworth, K. M. 1974, AJ, 79, 1384

Daub, C. T. 1982, ApJ, 260, 612

Durand, S., Acker, A., \& Zijlstra, A. 1998, A\&AS, 132, 13

Dutra, C. M., \& Maciel, W. J. 1990, RMA\&A, 21, 264

Fich, M., Blitz, L., \& Stark, A. A. 1989, ApJ, 342, 272

Fich, M., \& Tremaine, S. 1991, ARA\&A, 29, 409

Frink, S., Fuchs, B., Roser, S., \& Wielen, R. 1996, A\&A, 314, 430

Honma, M., \& Sofue, Y. 1997, PASJ, 49, 453

Hron, J. 1987 A\&A, 176, 34

Kingsburgh, R. L., \& Barlow, M. J. 1992, MNRAS, 257, 317

Kuijken, K., \& Tremaine, S. 1994, ApJ, 421, 178

Maciel, W. J. 1984, A\&AS, 55, 253

Maciel, W. J., \& Pottasch, S. R. 1980, A\&A, 88, 1

Mallik, D. C. V., \& Peimbert, M. 1988, RMA\&A, 16, 111

Mendez, R. H., Kudritzki, R. P., Herrero, A., Husfield, D., \& Groth, H. G. 1988, A\&A, 190, 113

Merrifield, M. R. 1992, AJ, 103, 1552

Metzger, M. R., \& Schechter, P. L. 1994, ApJ, 420, 177

Milne, D. K. 1982, MNRAS, 200, 51p

O'Dell, C. R. 1962, ApJ, 135, 371

Peimbert, M. 1990, RMA\&A, 20, 119

Pont, F., Mayor, M., \& Burki, G. 1994, A\&A, 285, 415

Pottasch, S. R. 1984, Planetary Nebulae (Reidel: Dordrecht)

Reid, M. J. 1993, ARA\&A, 31, 345

Rohlfs, K., Chini, R., Wink, J. E., \& Bohme, R. 1986, A\&A, 158, 181

Rohlfs, K., \& Kreitshmann, J. 1987. A\&A, 178, 95

Schneider, S. E., \& Terzian, Y. 1983, ApJ, 234, L61

Schneider, S. E., Terzian, Y., Purgathofer, A., \& Perinotto, M. 1984, ApJS, 52, 399

van de Steene, G. C., \& Zijlstra, A. A. 1994. A\&AS, 108, 485

Zhang, C. Y. 1995. ApJS, 98, 659 